\title{
Calculation of the different shielding properties of locally developed ilmenite-magnetite (I-M) concrete
}

\author{
Hossain Sahadath ${ }^{1}$, Abdus Sattar Mollah ${ }^{2}$, Khorshed Ahmad Kabir ${ }^{1}$ and Md. Fazlul Huq ${ }^{1, \star}$ \\ 1 Department of Nuclear Engineering, University of Dhaka Dhaka-1000, Bangladesh. \\ 2 Bangladesh Atomic Energy Commission, Bangladesh.
}

Received 28 November 2014 - Accepted 5 February 2015

\begin{abstract}
In this study the total atomic cross-section $\left(\sigma_{a}\right)$, total electronic cross-section $\left(\sigma_{e}\right)$, effective atomic number $\left(Z_{\text {eff }}\right)$ and effective electron density $\left(N_{\text {eff }}\right)$ of locally developed ilmenite-magnetite (I-M) concrete were calculated analytically for different photon energies from $1 \mathrm{keV}$ to $20 \mathrm{MeV}$ and compared with concretes of different densities and compositions. The effect of $Z_{\text {eff }}$ on the mass attenuation coefficient $\left(\mu / \rho, \sigma_{a}, \sigma_{e}\right)$ is shown graphically. The values of $\sigma_{a}$, $\sigma_{e}, Z_{\text {eff }}$ and $N_{\text {eff }}$ of I-M concrete were found to be higher than those of the ordinary concretes and in some cases than those of heavy concretes, which proved I-M concrete's shielding effectiveness. The results of this study will provide some useful information for a shielding material database for practical shielding calculation.
\end{abstract}

Keywords: atomic cross-section / electronic cross-section / effective atomic number / effective electron density

\section{Introduction}

To enable personnel to work in the vicinity of a nuclear installation, it is necessary to attenuate the radiation in a biological shield surrounding the radiation sources, so as to reduce the radiation dose to a tolerable level in the region beyond the shield. This is usually achieved by surrounding the radiation source with a sufficiently large mass of high-density material. Concrete is inexpensive and has structural strength as well as good shielding characteristics. In view of these advantages, concrete has been widely used as a shielding material. The most commonly used types of concretes are those based on barite, serpentine, steel magnetite, ordinary concrete, etc. The heavy concretes have proved themselves to be the most suitable materials for the attenuation of gamma radiation. At the beach sand processing plant in Cox's Bazar, in Bangladesh, ilmenite and magnetite sand are obtained in huge quantity as tailings. Investigation showed that these materials have great potential in the development of high-density concrete to be used as a shield. The ilmenite-magnetite (I-M) concrete is a heavy concrete produced locally with sand, stone chips and cement in the ratio 100:100:36 by volume, respectively. It has been used as the shield of the 3MW (Thermal) TRIGA Mark II Research Reactor at the Atomic Energy Research Establishment in Bangladesh. The gradation and composition of its aggregates are shown in Table 1 and the elemental composition is shown in Table 2. The knowledge of parameters such as the total atomic cross-section $\left(\sigma_{a}\right)$, total electronic cross-section $\left(\sigma_{e}\right)$, effective atomic number $\left(Z_{\text {eff }}\right)$ and effective electronic

\footnotetext{
^ Correspondence: fazlul.huq@du.ac.bd
}

Table 1. I-M concrete aggregates.

\begin{tabular}{|c|c|}
\hline \multicolumn{2}{|c|}{ Coarse aggregate [stone chip gradation (vol\%)] } \\
\hline \multicolumn{2}{|c|}{$3.81 \mathrm{~cm}$ down - 20 black (crusher) } \\
\hline \multicolumn{2}{|c|}{$2.54 \mathrm{~cm}$ down - 30 black (crusher) } \\
\hline \multicolumn{2}{|c|}{$1.91 \mathrm{~cm}$ down - 35 black (crusher) } \\
\hline \multicolumn{2}{|c|}{$1.27 \mathrm{~cm}$ down - 15 black (crusher) } \\
\hline \multicolumn{2}{|l|}{ Fine aggregate composition (vol\%) } \\
\hline Ordinary sand & 20 \\
\hline Ilmenite & 40 \\
\hline Magnetite & 40 \\
\hline Mix ratio (cement:sand:stone chips) & $36: 100: 100$ \\
\hline Net water/cement ratio & 0.5 \\
\hline Slump $(\mathrm{cm})$ & 1.27 \\
\hline
\end{tabular}

density $\left(N_{\text {eff }}\right)$ plays an important role in understanding the physical properties of any concrete. Scattering and absorption of X-ray and gamma radiation are related to the density and atomic number of an element. In composite materials, they are related to the effective atomic number and effective electron density.

Many experimental and theoretical studies have been performed on the total atomic cross-section, total electronic crosssection, effective atomic number and effective electronic density of different materials for different photon energies such as Au alloys (Han and Demir, 2010), Ti and Ni alloys (Han and Demir, 2009), carbon steel and stainless steels (Singh and Badiger, 2013), steel types 304 and 347 (Al-Jaff, 2013), polymers (Kucuk et al., 2013), alkali halides (Madhusudhan Rao et al., 2013), solid-state track detectors (Medhat, 2011), 
Table 2. Elemental composition of I-M concrete.

\begin{tabular}{|c|c|c|c|c|c|}
\hline Element & $\begin{array}{l}\text { Atomic } \\
\text { number }\end{array}$ & $\begin{array}{l}\text { Atomic mass } \\
(\mathrm{g})\end{array}$ & $\begin{array}{l}\text { Elemental density } \\
\qquad\left(\mathrm{g} \mathrm{cm}^{-3}\right)\end{array}$ & $\begin{array}{l}\text { Weight } \\
\text { fraction }\end{array}$ & $\begin{array}{l}\text { Total density } \\
\left(\mathrm{g} \mathrm{cm}^{-3}\right)\end{array}$ \\
\hline $\mathrm{H}$ & 1 & 1.008 & 0.0157 & 0.005647 & \\
\hline $\mathrm{C}$ & 6 & 12.011 & 0.0022 & 0.000791 & \\
\hline $\mathrm{O}$ & 8 & 16.000 & 1.0523 & 0.378525 & \\
\hline $\mathrm{Mg}$ & 12 & 24.320 & 0.1014 & 0.036474 & \\
\hline $\mathrm{Al}$ & 13 & 26.980 & 0.0497 & 0.017877 & \\
\hline $\mathrm{Si}$ & 14 & 28.090 & 0.1349 & 0.048525 & \\
\hline $\mathrm{P}$ & 15 & 30.975 & 0.0002 & 0.000071 & \\
\hline S & 16 & 32.066 & 0.0016 & 0.000575 & 2.78 \\
\hline $\mathrm{Ca}$ & 20 & 40.080 & 0.2469 & 0.088812 & \\
\hline $\mathrm{Ti}$ & 22 & 47.900 & 0.3563 & 0.128165 & \\
\hline V & 23 & 50.950 & 0.0021 & 0.000755 & \\
\hline $\mathrm{Cr}$ & 24 & 52.010 & 0.0010 & 0.000359 & \\
\hline Mn & 25 & 54.940 & 0.0084 & 0.003021 & \\
\hline $\mathrm{Fe}$ & 26 & 55.850 & 0.7863 & 0.282841 & \\
\hline $\mathrm{Ni}$ & 28 & 58.710 & 0.0012 & 0.000431 & \\
\hline
\end{tabular}

Table 3. Comparison of the half-value layer (HVL) of different concretes.

\begin{tabular}{cccccc}
\hline $\begin{array}{c}\text { Photon } \\
\text { energy (MeV) }\end{array}$ & I-M & Ordinary-1 & $\begin{array}{c}\text { Half-value layer (atom } \mathbf{~ c m}^{\mathbf{2}} \text { ) } \\
\text { Concrete type } \\
\text { Ordinary-2 }\end{array}$ & Barite & Serpentine \\
\hline 6.000 & 0.415 & 0.720 & 0.656 & 0.154 & 0.776 \\
1.000 & 0.436 & 0.790 & 0.714 & 0.149 & 0.851 \\
10.000 & 0.443 & 0.834 & 0.750 & 0.143 & 0.900 \\
12.000 & 0.443 & 0.856 & 0.766 & 0.138 & 0.924 \\
15.000 & 0.443 & 0.890 & 0.791 & 0.130 & 0.961 \\
18.000 & 0.437 & 0.900 & 0.797 & 0.125 & 0.973 \\
20.000 & 0.433 & 0.907 & 0.800 & 0.121 & 0.980 \\
\hline
\end{tabular}

concrete materials (Y1lmaz et al., 2011; El-Khayatt and Akkurt, 2013), etc. There have been some experimental and theoretical investigations to determine the neutron-shielding properties of I-M (Bhuiyan et al., 1991; Ahmed et al., 1992, 1999). There are almost no reports on the study of $\sigma_{a}, \sigma_{e}, Z_{e f f}$ and $N_{\text {eff }}$ of this locally developed concrete for different photon energies. This prompted us to carry out this work. The objective of this study is to generate a database for $\sigma_{a}, \sigma_{e}, Z_{e f f}$ and $N_{\text {eff }}$ of this locally developed concrete, which will be helpful for practical shielding calculation.

\section{Theoretical background and calculation}

\subsection{Total atomic cross-section $\left(\sigma_{a}\right)$ and electronic cross-section $\left(\sigma_{e}\right)$}

The atomic attenuation coefficient $\left(\sigma_{a}\right)$ is the fraction of an incident gamma-ray beam that is attenuated by a single atom. Another way of saying the same thing is that the atomic attenuation coefficient is the probability that an absorber atom will interact with one of the photons in the beam. Note that the dimensions of $\sigma_{a}$ are $\mathrm{cm}^{2}$, the units of area. For this reason, the atomic attenuation coefficient is almost always referred to as the cross-section of the absorber. The unit in which the crosssection is specified is the barn, $b$.

The electronic attenuation coefficient is the fraction of an incident gamma-ray beam that is attenuated by a single electron. The electronic attenuation coefficient is also called the electronic cross-section and is symbolized by $\sigma_{e}$. The total atomic cross-section $\sigma_{a}$ and the total electronic cross-section $\sigma_{e}$ can be obtained by the following formulas:

$$
\begin{aligned}
\sigma_{\mathrm{a}} & =\frac{1}{\mathrm{~N}_{\mathrm{A}}} \sum_{i} f_{i} A_{i}\left(\frac{\mu}{\rho}\right)_{i} \\
\sigma_{e} & =\frac{1}{N_{A}} \sum_{i} f_{i} \frac{A_{i}}{Z_{i}}\left(\frac{\mu}{\rho}\right)_{i}
\end{aligned}
$$

where $f_{i}$ is the fractional abundance of element $i$ in the mixture; $Z_{i} A_{i}$ are the atomic number and atomic mass, respectively, $\left(\frac{\mu}{\rho}\right)_{i}$ is the total mass attenuation coefficient of the $i$ th element in the mixture and $N_{A}$ is Avogadro's number.

\subsection{The effective atomic number $\left(Z_{\text {eff }}\right)$ and effective electron density $\left(N_{\text {eff }}\right)$}

The effective atomic number of a material is the atomic number of a hypothetical element that attenuates photons at the same rate as the material. In composite material, a single number cannot represent the atomic number uniquely across the entire energy range, as the partial interaction cross-sections have different atomic number dependence. The effective atomic 
numbers of the concrete samples were obtained by means of the practical formula (El-Khayatt and Akkurt, 2013)

$$
Z_{\text {eff }}=\frac{\sum_{i} f_{i} A_{i}\left(\frac{\mu}{\rho}\right)_{i}}{\sum_{i} f_{i} \frac{A_{i}}{Z_{i}}\left(\frac{\mu}{\rho}\right)_{i}}
$$

The effective electron density, expressed in number of electrons per unit mass, is closely related to the effective atomic number and given by

$$
N_{e f f}=N_{A} \frac{Z_{\text {eff }}}{\langle A\rangle} .
$$

\subsection{Half-value layer (HVL)}

The half-value layer (HVL) is defined as the thickness of a shield or an absorber that reduces the radiation level by a factor of 2; that is, to half the initial level. (The HVL is also called a half-value thickness.) In this study, the HVL was calculated in units of $\left(\right.$ atom $\left./ \mathrm{cm}^{2}\right)$ using the atomic cross-section $\sigma_{a}$ by the following equation:

$$
x_{\frac{1}{2}}=H V L=\frac{0.693}{\sigma_{a}} .
$$

The mass attenuation coefficients for the elements at different photon energies were taken from Hubbell and Seltzer (1995). For comparisons, the shielding parameters for other types of concretes were calculated with the same method as for I-M concrete. The composition of barite, serpentine and ordinary concrete-1 was taken from Sharifi et al. (2013) and ordinary concrete-2 from Cember and Jonson (2009).

\section{Results and discussions}

\subsection{Total atomic cross-section}

The variation of the total atomic cross-section $\sigma_{a}$ (barn/atom) of I-M concrete with photon energy is shown in Figure 1 and compared with concretes of different densities and compositions. In the low-energy range from a few $\mathrm{keV}$ to about $100 \mathrm{keV}$, the $\sigma_{a}$ (barn/atom) for I-M concrete is higher than that of the ordinary concretes and serpentine. This is due to the higher values of mass attenuation coefficients of the elements of I-M concrete, which can be explained by the dominance of the photoelectric effect in this energy range. Since the photoelectric effect strongly depends on the atomic number of the element we can say that materials containing elements of higher atomic number will have a higher atomic cross-section. This is exactly what we can see from Figure 1. When the photon energy is exactly equal to the binding energy of the innermost $\mathrm{K}$ or $\mathrm{L}$ shell electron in the elements, $\sigma_{a}$ (barn/atom) shows a peak in the curve known as the photoelectric edge. The sharp decrease in $\sigma_{a}$ (barn/atom) is due to the energy dependence of the photoelectric effect.

In the intermediate energy region where Compton scattering is dominant, there is a smooth fall in the curve because this interaction depends inversely on photon energy. The $\sigma_{a}$ (barn/atom) of I-M concrete is also found to be higher

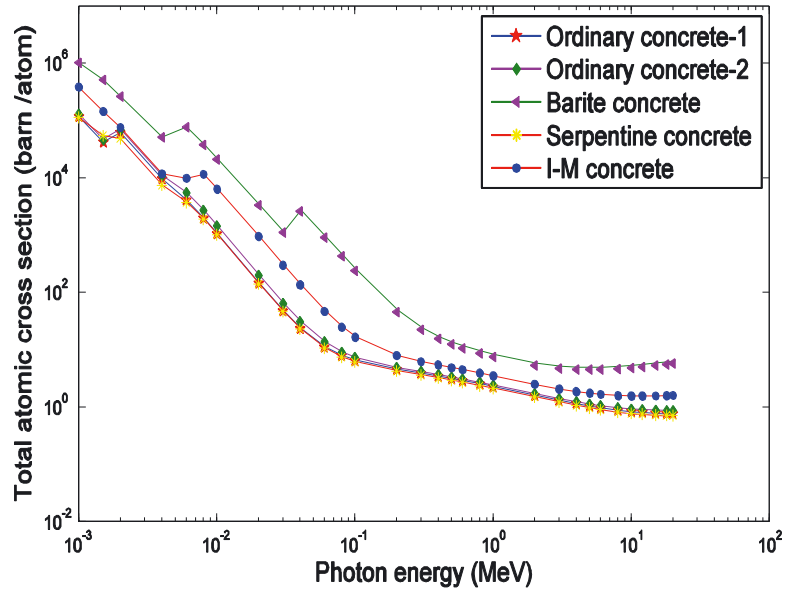

Figure 1. Variation of the total atomic cross-section with photon energy.

than that of the other concretes except barite, which contains $46.34 \%$ barium. Although serpentine has a higher effective electron density than I-M concrete, the elements of higher atomic masses in I-M concrete compensated for the effect of its lower electron density in this energy range. In the higherenergy region greater than $10 \mathrm{MeV}$, the probability of pair production increases with photon energy, which is shown by the smooth rise in the curve at the end. Also, in this region $\sigma_{a}$ (barn/atom) of I-M concrete is higher than that of the serpentine and ordinary concretes. This can also be explained by the elements of higher atomic number in I-M concrete.

\subsection{Total electronic cross-section $\left(\sigma_{e}\right)$}

The total electronic cross-section $\sigma_{e}$ (barn/electron) of any material at a particular energy depends mainly on the mass attenuation coefficient of the elements in the material at that particular energy. The ratio of atomic mass to atomic number does not have any significant effect on the electronic crosssection since it is nearly constant for all the elements in the periodic table except for the higher atomic number elements. However, the higher atomic number elements are favored by photoelectric absorption and pair production. The variation of $\sigma_{e}$ (barn/electron) of different concretes with photon energy is shown in Figure 2. It is seen from the figure that $\sigma_{e}$ (barn/electron) of I-M concrete is much higher than that of the ordinary concretes and serpentine in the low-energy region of up to $0.1 \mathrm{MeV}$ where photoelectric absorption dominates the interaction process. This is because of the higher $(\mu / \rho)$ of the elements of I-M concrete. It is also seen from the figure that photoelectric absorption dominates over a greater energy range in the higher effective atomic number material than in the lower one. Peaks in the curve and the sharp decrease in $\sigma_{e}$ (barn/electron) are also the consequences of photoelectric absorption. In the energy range from $0.5 \mathrm{MeV}$ to $3 \mathrm{MeV}$, Compton scattering is dominant in all the materials and $\sigma_{e}$ (barn/electron) is nearly the same for all materials irrespective of their effective atomic number. Moreover, the ratio $A / Z$ is nearly constant for all the elements. Therefore, $\sigma_{e}$ is the same for all materials at a particular energy. As energy increases beyond this region pair production starts to dominate, 


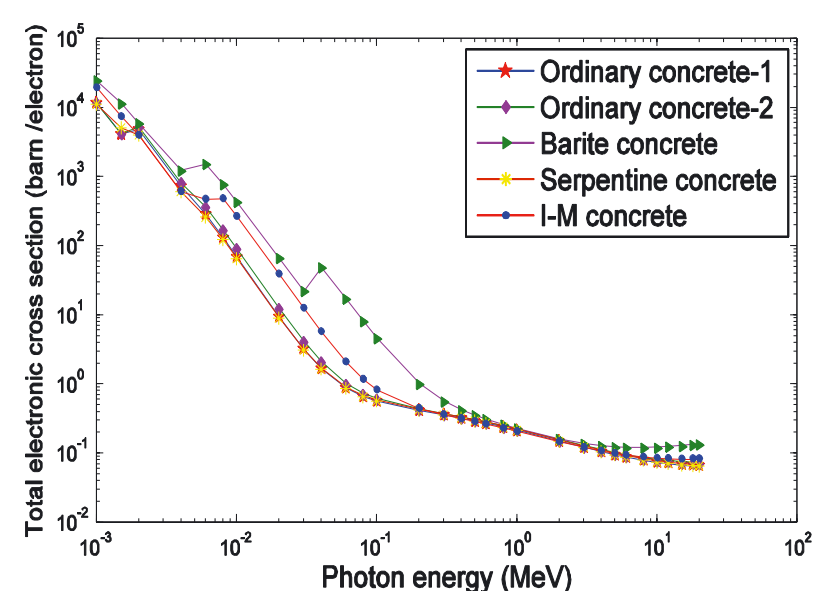

Figure 2. Variation of the total electronic cross-section with photon energy.

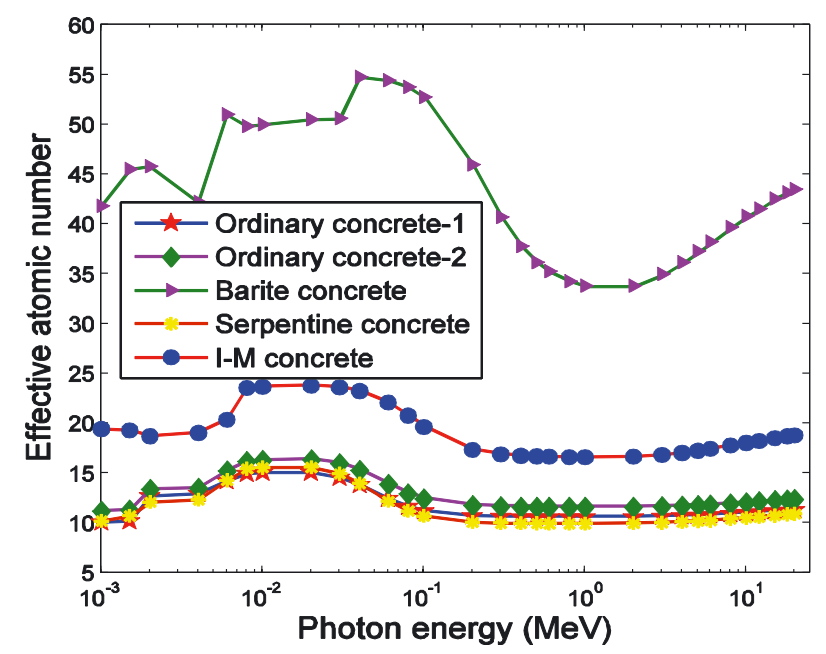

Figure 3. Variation of the effective atomic number with photon energy.

whose probability increases with both the photon energy and the effective atomic number of the material. Therefore, $\sigma_{e}$ of the I-M concrete is higher than that of the other concretes except barite, which has the highest effective atomic number.

\subsection{Effective atomic number $\left(Z_{\text {eff }}\right)$ and effective electron density $\left(N_{e f f}\right)$}

The effective atomic number of the I-M concrete at different photon energies is compared with other types of concretes in Figure 3. It is clear from the figure that I-M concrete has higher $Z_{\text {eff }}$ values than the ordinary concretes and serpentine due to its higher atomic number elements. Barite, which contains nearly fifty percent barium, has the highest value of effective atomic number. The minimum $Z_{\text {eff }}$ values were found in the intermediate region, where Compton scattering is dominant, which is independent of atomic number and approximately equal to the mean atomic number of the samples. The maximum value of $Z_{\text {eff }}$ was found in the low-energy range, where photoelectric absorption is dominant. Figure 4 presents the variations of effective electron density $\left(N_{\text {eff }}\right)$ of different



Figure 4. Variation of the effective electron density with photon energy.

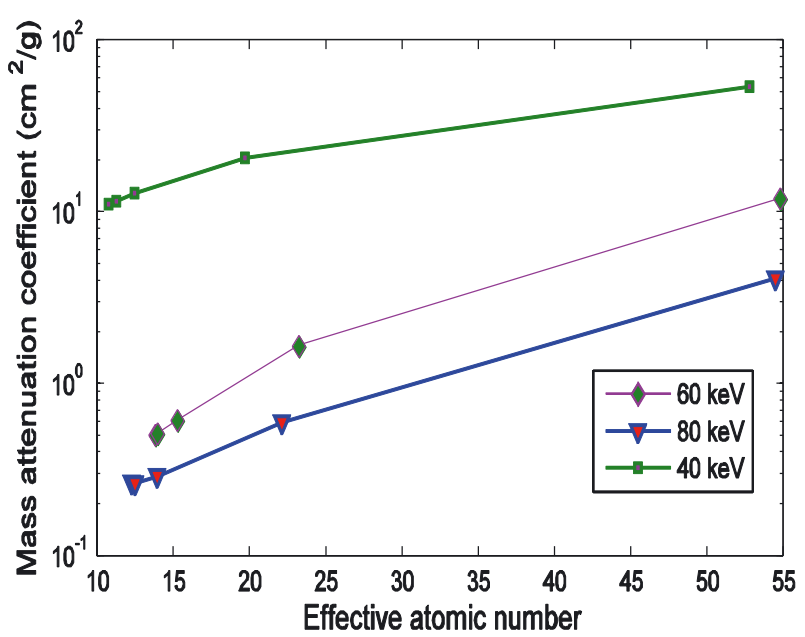

Figure 5. Variation of the mass attenuation coefficient with $Z_{\text {eff }}$.

concretes at photon energies from $1 \mathrm{keV}$ to $20 \mathrm{MeV}$. Peaks in the curves indicate the photoelectric edge. Since incident photons interact with individual electrons, therefore higher $N_{\text {eff }}$ means higher attenuation of photons. The effective electron density of I-M concrete is higher than that of the ordinary concretes and serpentine in the energy range where photoelectric absorption and pair production are dominant. In the intermediate energy region where Compton scattering is dominant, serpentine is slightly better than I-M concrete for its higher effective electron density. Figure 5 shows the variation of the total mass attenuation coefficient $(\mu / \rho)$ with $Z_{\text {eff }}$ at low photon energies where photoelectric absorption is dominant. It is clear from Figure 5 that the $\mu / \rho$ at these energies strongly depends on $Z_{\text {eff }}$ and increases with $Z_{\text {eff }}$. Figures 6 and 7 depict the dependence of the total electronic cross-section $\left(\sigma_{e}\right)$ and total atomic cross-section $\left(\sigma_{a}\right)$ on $Z_{e f f}$, respectively. The variations observed were similar to those the case of $\mu / \rho$. Therefore, the concretes with higher $Z_{e f f}$ will have higher $\sigma_{a}$ and $\sigma_{e}$ at these low energies where photoelectric absorption is dominant. Also, the higher the $\sigma_{a}$ and $\sigma_{e}$, the better the shielding will be. 


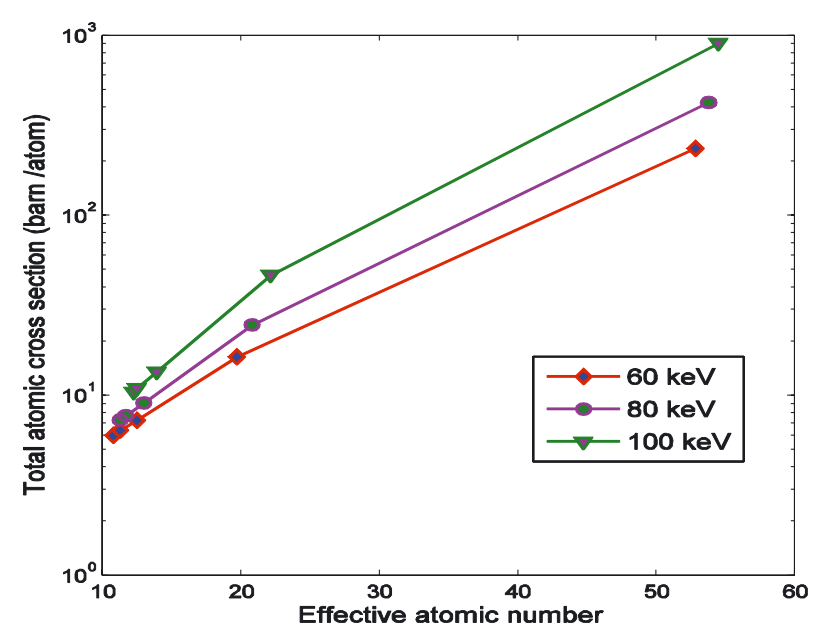

Figure 6. Variation of the total atomic cross-section with $Z_{\text {eff }}$.

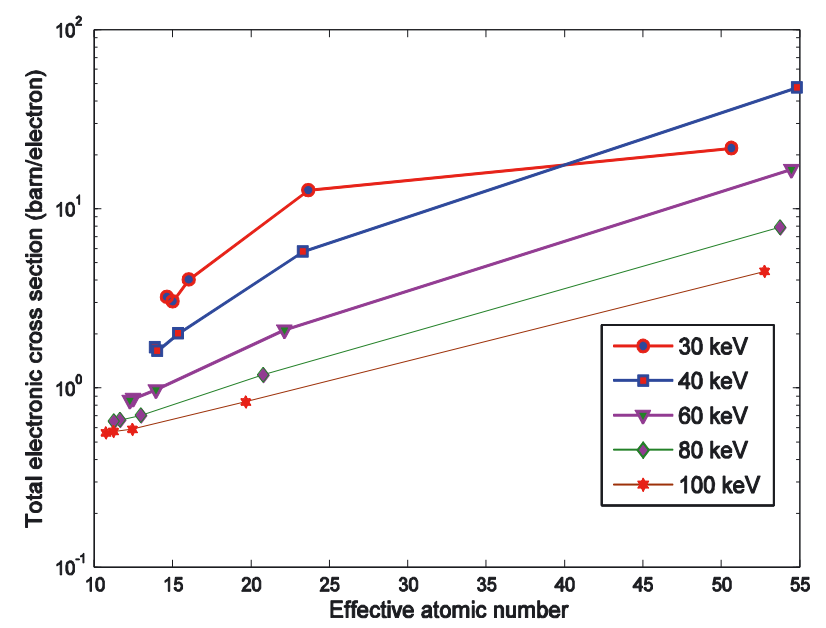

Figure 7. Variation of the total electronic cross-section with $Z_{e f f}$.

\section{Conclusions}

The atomic cross-section, electronic cross-section, effective atomic number and effective electron density calculated in this study reflect the good quality of I-M concrete as far as its shielding properties are concerned. The effective electron density $\left(N_{\text {eff }}\right)$ and effective atomic number $\left(Z_{\text {eff }}\right)$ are closely related and they have the same qualitative energy dependence. The mass attenuation coefficient $(\mu / \rho)$ of the element is a useful and sensitive physical quantity to determine the $\left(Z_{\text {eff }}\right)$ and $\left(N_{\text {eff }}\right)$ of a concrete. The results of this study will provide a database for these parameters for this locally developed concrete as well as for other types of concretes, which will facilitate the shielding design problem. It can be concluded that I-M concrete is superior to ordinary concretes in the whole energy range and better than serpentine in the region where the photoelectric effect and pair production are dominant. Since raw materials are locally available, I-M concrete will be an excellent, cost-effective and less space-consuming shielding material against gamma rays. It can be used as a shield for reactors, accelerators, X-ray installation and other hot laboratories, as well as commercial gamma-ray irradiation facilities.

\section{References}

Ahmed F.U., Bhuiyan S.I., Mollah A.S, Rahman M.M. (1992) Measurement of gamma ray shielding properties of ilmenitemagnetite concrete and polyborn slabs using a ${ }^{252} \mathrm{Cf}$ source, Nuclear Technology 98, 379-386.

Ahmed F.U., Bhuiyan S.I., Mollah A.S, Sarder M.R., Huda M.Q., Rahman M., Mondal M.A.W. (1999) Studies on the shielding properties of polyboron and ilmenite-magnetite concrete using a reactor beam, Nuclear Technology 126,196-204.

Al-Jaff S.M.A.A. (2013) Investigation the Effective atomic number, electron density, Half value layer and mean free path of steel types 304 and 347 in the energy range $40 \mathrm{KeV}-130 \mathrm{KeV}$, Journal of Natural Sciences Research 3,15.

Bhuiyan S.I., Ahmed F.U., Mollah A.S., Rahman M.A. (1991) Studies of neutron shielding properties of ilmenite- magnetite concrete using a ${ }^{252} \mathrm{Cf}$ source, Nuclear Technology 93, 357-361.

Cember H., Jonson E. (2009) Introduction to Health Physics, fourth ed. Chapt. 5, Department of Environmental and Radiological Health Sciences Colorado State University.

El-Khayatt A.M., Akkurt I. (2013) Photon interaction, energy absorption and neutron removal cross section of concrete including marble, Ann. Nucl. Energy 60, 814.

Han I., Demir L. (2009) Mass attenuation coefficients, effective atomic and electron numbers of $\mathrm{Ti}$ and $\mathrm{Ni}$ alloys, Radiat. Meas. 44, 289-294.

Han I., and Demir L. (2010) Studies on the effective atomic number, electron densities and mass attenuation coefficients in Au alloys, Journal of X-Ray Science and Technology 18, 39-46.

Hubbell J.H., Seltzer S.M. (1995) Tables of X-ray Mass Attenuation Coefficients and Mass Energy-Absorption Coefficients $1 \mathrm{keV}$ to $20 \mathrm{MeV}$ for Elements $\mathrm{Z}=1$ to 92 and 48 Additional Substances of Dosimetric Interest. NISTIR-5632, National Institute of Standards and Technology, Gaithersburg.

Kucuk N., Cakir M., Isitman N.A. (2013) Mass Attenuation Coefficients, Effective Atomic Numbers And Effective Electron Densities For Some Polymers, Radiat. Prot. Dosim.153, 127-134.

Madhusudhan Rao A.S., Narender K., Gopal Kishan Rao K., Gopi Krishna N., Radha Krishna Murthy (2013) Mass Attenuation Coefficients, Effective atomic and Electron Numbers of Alkali Halides for Multi-Energetic Photons, Research Journal of Physical Sciences 1 (6), 11-16.

Medhat M.E. (2011) Studies on effective atomic numbers and electron densities in different solid state track detectors in the energy range $1 \mathrm{keV}-100 \mathrm{GeV}$, Ann. Nucl. Energy 38, 1252-1263.

Sharifi Sh., Bagheri R., Shirmardi S.P. (2013) Comparison of shielding properties for ordinary, barite, serpentine and steel magnetite concretes using MCNP-4C code and available experimental results, Ann. Nucl. Energy 53, 529-534.

Singh V.P., Badiger N.M. (2013) Study of mass attenuation coefficients, effective atomic numbers and electron densities of carbon steel and stainless steels, Radioprotection 48, 431-443.

Yılmaz E., Baltas H., Kırıs E., Ustabas I., Cevik U., El-Khayatt A.M. (2011) Gamma ray and neutron shielding properties of some concrete materials, Ann. Nucl. Energy 38, 2204-2222. 